\title{
Tendencias en la Búsqueda de la Verdad
}

Este comentario está disponible en www.anuariocdh.uchile.cl

\author{
Eduardo González Cueva*
}

\section{Introducción}

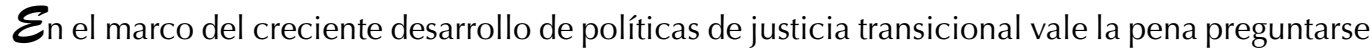
por los procesos que ocurren en el terreno de la búsqueda de la verdad. El interés en las comisiones de la verdad continúa siendo muy fuerte en transiciones políticas alrededor del mundo y los puntos de referencia históricos -Chile y Sudáfrica- siguen estando presentes cuando actores políticos y sociales confrontan legados de violencia y autoritarismo.

Ahora bien, diversas señales de la práctica reciente parecen indicar que nuevos ejercicios de diseño y ejercicio de búsqueda de la verdad intentan ir más allá de los mitos fundacionales que estuvieron detrás de comisiones previas. Ha pasado, así, el momento en que las comisiones se percibían como una medida provisoria, un instrumento para ganar tiempo en ausencia de "justicia real": hoy se reconoce con mayor claridad que la búsqueda de la verdad es un valor en sí, no menor al de esclarecer responsabilidades penales. Pero -tal vez- está pasando también el momento en que las comisiones se ven como un instrumento de potencial casi ilimitado, del que se exige el milagro político de suprimir completamente los efectos del pasado por vía de la reconciliación automática.

Naturalmente, sería injusto reducir el legado de las comisiones chilena y sudafricana a tales simplificaciones, pero es innegable que su apropiación -particularmente por élites políticas posttransición- ha tendido a la unilateralidad. Son esos enfoques unilaterales los que están mostrando sus limitaciones $y$, aunque inventariar algunos hechos recientes no puede de ningún modo ilustrar una tendencia irreversible, vale la pena identificar ciertos elementos en común.

\section{Dos generaciones de comisiones de la verdad}

Un cuarto de siglo luego de la experiencia de la Comisión Nacional sobre la Desaparición Forzada de Personas (CONADEP) en Argentina, las comisiones de la verdad continúan ejerciendo una clara atracción sobre los actores políticos activos en sociedades en transición política. Instancias internacionales han identificado principios básicos ${ }^{1}$ que guían su actuación en contextos de superación de conflictos o autoritarismo, a la vez que actores domésticos invocan con frecuen-

\footnotetext{
* Sociólogo peruano (egonzalez@ictj.org); Ex miembro del equipo profesional de la Comisión de la Verdad y Reconciliación del Perú y Asociado principal del Centro Internacional por la Justicia Transicional (ICTJ).

1 "Conjunto de principios actualizados para la lucha contra la impunidad", Informe de la experta independiente Diane Orentlicher a la Comisión de Derechos Humanos de la ONU, E/CN.4/2005/102/Add.1; "El derecho a la verdad", Resolución № 2005/66 de la Comisión de Derechos Humanos de la ONU; "El estado de derecho y la justicia de transición en las sociedades que sufren o han sufrido conflictos", Informe del Secretario General de la ONU, S/2004/616, 3 de agosto de 2004.
} 
cia el éxito de comisiones de la verdad en otros países como política posible para cimentar la reconciliación nacional.

Sin embargo, y pese a los avances normativos, consideraciones pragmáticas de política siguen determinando la aceptación de estos instrumentos. Con alguna frecuencia es posible detectar que los actores políticos encuentran en las comisiones sencillamente una alternativa a mecanismos judiciales de establecimiento de responsabilidades, ya sea bajo la ilusión de posponerlos o evitarlos completamente.

Esas ilusiones tienen un doble carácter: por un lado está la expectativa de los actores políticos y, por otro, la respuesta de los actores sociales. Las expectativas políticas han sido una y otra vez confrontadas por los procesos reales de instituciones que hicieron valer su independencia, interpretaron creativamente sus mandatos y sentaron las bases para identificar principios básicos y lecciones aprendidas.

La ilusión política de una respuesta no traumática al legado autoritario de impunidad tiene una historia y varias fases. La fase preliminar, que ni siquiera habría que numerar puesto que precede a la creación de comisiones de la verdad, está marcada por las transiciones del sur de Europa en los años 70, donde la dicotomía "recordar u olvidar" fue clara. El interés político determinó que -de esas experiencias- se recordara la transición española, con el aparente éxito de su política de amnesia oficial, y se ignorara la experiencia griega, que culminó en los juicios a los líderes de la junta militar de ese país.

La primera fase propiamente dicha de las comisiones de la verdad tuvo lugar durante la siguiente oleada democratizadora, esta vez en América Latina durante los años ochenta y tempranos noventa. Dichas transiciones se percibían como frágiles e inseguras, lo que incentivaba el interés en España (cercana, además, por razones culturales). Pero, al mismo tiempo, eran transiciones de países con violencias recientes, por lo que no bastaba con el ejemplo español donde el transcurso del tiempo parecía haber debilitado los reclamos de las víctimas.

En esa fase -latinoamericana- se crea una "primera generación" de comisiones en las que implícitamente se advierte la esperanza de lograr alguna discrecionalidad en el manejo de los tiempos políticos. El trabajo de una comisión de la verdad durante la transición podía ganar el tiempo necesario para normalizar el poder democrático, debilitar los poderes fácticos y crear mejores condiciones para una potencial fase penal de la lucha contra la impunidad. Considerando el ambiente de impunidad de facto o de jure en que operaron, se advierte en comisiones como la chilena, la salvadoreña o la guatemalteca un diseño que intenta cumplir -por vías diferentes- con un mandato de esclarecimiento que no menoscabe las posibilidades de futuros procesos penales.

En una segunda fase -ligada a transiciones africanas y asiáticas- se detecta el esfuerzo de encontrar una vía alternativa de resolución de conflictos, que evite completamente el proceso judicial. Se exploran entonces formas de enriquecer la investigación del pasado con mecanismos de justicia tradicional menos enfocados en el castigo que en el fortalecimiento de lazos comunitarios y -por consiguiente- en formas de mediación entre víctima y victimario. La decisión sudafricana de ofrecer amnistías condicionales a quienes cooperasen plenamente con el esclarecimiento de los crímenes en los que hubieran tenido participación abrió este "segundo momento", en el que el diseño de las comisiones busca alterar el marco en el que actúan los mecanismos jurisdiccionales, al menos en el plano doméstico. El drama y visibilidad de las audiencias públicas presididas por Desmond Tutu capturaron la imaginación mundial y -como suele ocurrir con la imaginación-mistificaron los procedimientos legales puestos en obra por el diseño sudafricano como la traducción de legados ancestrales o principios religiosos fundamentales aplicables universalmente. Múltiples esfuerzos 
posteriores -que podríamos Ilamar una "segunda generación" de comisiones de la verdad- han intentado aplicar total o parcialmente el modelo sudafricano.

Estos momentos en la práctica de las comisiones evocan mitos e imágenes fundacionales: la transición chilena, que desnuda poco a poco la verdad sobre la dictadura erosionando sus aún significativas parcelas de poder, y la transición sudafricana, que inspira a todas las partes a pedir y otorgar perdón. Cualquiera de los dos mitos es -por cierto- un avance en la lucha contra la impunidad, si se los compara con el mito anterior, de la España postfranquista que establece un pacto de silencio. Pero ambos -en algunas lecturas- pueden conducir a expectativas irreales que ningún instrumento de política puede solventar: consolar universalmente a las víctimas, calmar los ánimos de las instituciones en que operan los victimarios y conducir la nación al perdón.

Las lecciones aprendidas y los principios descubiertos a partir de las "generaciones" mencionadas han superado con creces las ilusiones faltas de realismo. De hecho, la práctica de algunas comisiones recientes parece indicar que hemos llegado a los límites de la "segunda generación" y avanzamos hacia una nueva síntesis de problemas, retos y -seguramente- ilusiones.

Dos señales revelan una tendencia, al menos tentativa: (a) la disputa sobre los legados de recientes comisiones de la verdad, que cuestionan la presunción de su poder como instrumentos automáticos de reconciliación y (b) el fracaso de esfuerzos que sacrificaban claramente los derechos de las víctimas. Es imposible predecir si estamos ante una naciente "tercera generación" de comisiones de la verdad, pero vale la pena repasar-aunque fuese a modo de inventario- algunos desarrollos actuales.

\section{Legados en disputa}

El primer elemento que debe notarse en este balance es la insatisfacción generada por la promesa poco realista de un instrumento de completa eficacia para conjurar los problemas del pasado. La experiencia de la propia Sudáfrica, a más de diez años de su ejemplar comisión, debería inspirar una mirada sobria sobre las reales posibilidades de la búsqueda de la verdad como política de justicia transicional. Otros casos, como el de Sierra Leona y Timor Oriental, muestran a las claras el choque entre las expectativas de los actores políticos y el ejercicio de las comisiones, forzadas por las circunstancias a ir más allá de la ilusión de reconciliación automática.

\section{Sudáfrica}

Para los muchos diseminadores de la "ilusión sudafricana", el hecho que muchos Ex Comisionados y amplios sectores de víctimas muestren su profundo descontento con el ejercicio de la Comisión de la Verdad y Reconciliación, puede ser sorprendente. Pero esa sensación invade la actualidad de Sudáfrica: durante el año 2006, al conmemorarse una década de la entrega del informe final de la CVR, se vivieron intensos balances y debates en el país. Dos elementos se destacaron de manera particular: la decepción de grupos de víctimas y defensores de derechos humanos sobre la política de reparaciones recomendada por la CVR y la ansiedad ante la falta de progreso en investigaciones judiciales contra perpetradores que no recibieron el beneficio de una amnistía por parte de la CVR o que nunca la solicitaron ante ella.

En primer lugar, la política de reparaciones recomendada por la Comisión sudafricana no fue seguida por el gobierno. Éste rechazó la noción de un impuesto de solidaridad de empresas e individuos de muy altos ingresos para pagar reparaciones a las víctimas. Además, condenó las solicitudes que algunos sectores de víctimas elevaron a cortes de terceros países para buscar reparaciones 
de empresas que se beneficiaron con el régimen de Apartheid². La asociación "Khulumani", en representación de más de 40.000 víctimas, ha seguido desde 2002 una demanda en cortes estadounidenses contra diversas empresas multinacionales; pero el gobierno del Sr. Thabo Mbeki argumenta que este tipo de recursos aliena a las empresas privadas cuyas inversiones son necesarias para la economía sudafricana. Por ello, ha rendido testimonio en contra de la demanda ${ }^{3}$.

La resistencia del gobierno del Congreso Nacional Africano a otorgar reparaciones adecuadas a las víctimas identificadas por la CVR es particularmente dolorosa en un país donde -pese a la transformación del poder político- el legado del Apartheid es claro en la marginación económica sufrida por la mayoría de la población de color.

El propio arzobispo Tutu se ha quejado amargamente de la falta de disposición del gobierno de su país para otorgar reparaciones apropiadas a las cerca de 22.000 víctimas identificadas por la Comisión. En retrospectiva, el arzobispo ha expresado que la CVR debió haber otorgado reparaciones con el mismo carácter inmediato con el que otorgó amnistías ${ }^{4}$.

Por otro lado, vale la pena examinar el muy problemático intento sudafricano de continuar negando el acceso a la justicia penal a las víctimas. Como se sabe, la CVR sudafricana construyó su fórmula de amnistías condicionales sobre la base de una promesa política muy clara: ella sería un momento único en la historia del país. Los perpetradores que no aprovechasen su oportunidad de colaborar con la reconstrucción de los hechos no tendrían una nueva ocasión de hacerlo. De otro modo, si la posibilidad de obtener amnistía por colaboración hubiera sido permanente, se hubiera perdido el estímulo sobre los perpetradores, pues estos no habrían tenido sino que esperar a ver si la justicia estaba interesada en ellos para decidir, en cada caso, si colaborar. Además, la CVR contaba con el temor a la justicia penal para incentivar la completa colaboración de los perpetradores, puesto que nada sino un perfecto reconocimiento de los hechos podía garantizar una amnistía.

Sin embargo, en diversas ocasiones, Sudáfrica ha vaciado de significado la promesa de la CVR o ha amenazado con hacerlo. En 2002, el gobierno del Sr. Thabo Mbeki otorgó perdones a decenas de personas en prisión por hechos violentos cometidos durante el conflicto anti-apartheid, incluyendo varios cuya amnistía había sido denegada por la CVR ${ }^{5}$. Entre 2004 y 2006, activistas de derechos humanos han denunciado en numerosas ocasiones la intención de la fiscalía sudafricana de atribuirse el poder de negociar en privado nuevas amnistías con perpetradores que acepten colaborar con el esclarecimiento de los hechos, incluyendo aquellos que no lo hicieron en la época de la CVR 6 . La objeción es clara: si el fiscal puede revivir una y otra vez el mecanismo, ¿dónde deja eso a quienes sí cumplieron con el dramático ritual de admitir en público los más graves crímenes? ¿Qué garantía tienen las víctimas en estos nuevos casos de oponerse a la amnistía o de -por lo menos- escuchar la pública admisión del crimen?

\footnotetext{
2 Valji, Nahla, "South Africa: No Justice Without Reparation" en Open Democracy, 2003, versión digital en http://www. opendemocracy.net/debates/article.jsp?id=5\&debateld=130\&articleld=1326.

3 Reynolds, Norman, "Khulumani's Reparations Case and the Future of Human Rights", 2005, versión digital en http://www. khulumani.net/content/view/1589/13/.

4 Mail and Guardian, "Tutu Voices Fears Over Apartheid Prosecutions", abril de 2006, versión digital en http://www. khulumani.net/content/view/622/162/.

5 BBC, "South Africa Amnesty Raises Storm", 19 de mayo de 2002, versión digital en http://news.bbc.co.uk/2/hi/africa/1997262. stm.

6 Terreblanche, Christine, "Back Door Amnesty Revealed", 16 de noviembre de 2005, versión digital en http://www.int. iol.co.za/index.php?set_id=1\&click_id=13\&art_id=vn20051127070029402C565763.
} 
Uno de los elementos más problemáticos de la intención de los fiscales sudafricanos de utilizar una amnistía "por la puerta trasera" es la clara apariencia de sujeción a la voluntad política del gobierno. Algunos han denunciado que los nuevos criterios de la fiscalía emergieron al conocerse la posibilidad de juicios contra antiguos luchadores anti-apartheid que no obtuvieron amnistías de la CVR. La amnistía original fue justificada en numerosas ocasiones como un sacrificio necesario para evitar la posibilidad de una venganza política de los aún poderosos sectores racistas, mientras que la nueva amnistía no tendría más justificación que favorecer a los actuales poderosos.

De modo pues que en la misma Sudáfrica, donde tuvo lugar el más celebrado experimento de reconciliación por vía de la verdad, dos elementos clave del diseño de justicia transicional siguen estando ausentes: ni se ha ejercido la promesa de justicia contra quienes no cumplieron con revelar la verdad, ni se ha compensado adecuadamente a las víctimas que no obtuvieron remedio judicial.

\section{Sierra Leona}

No es exagerado decir que la Comisión de la Verdad y Reconciliación de Sierra Leona fue establecida sobre la base de la fuerte influencia de la comisión sudafricana. En efecto, esta CVR fue establecida por medio del tratado de paz de Lomé en 1999, que logró la paz con la guerrilla del "Frente Revolucionario Unido" de Foday Sankoh, otorgando a los rebeldes una amnistía general y puestos de gobierno al más alto nivel. La CVR, diseñada en ese contexto, debía "crear un clima que favorezca el intercambio productivo entre víctimas y victimarios", permitiendo a las primeras expresar sus sufrimientos y a los últimos "contar sus experiencias"7.

La Comisión, sin embargo, no empezó a trabajar de acuerdo a lo previsto porque la garantía de impunidad para Sankoh y su grupo no logró el objetivo prometido. El conflicto se reinició por la resistencia de los rebeldes a las funciones de mantenimiento de la paz de la ONU. Recién en mayo de 2000, luego de una intervención británica que culminó en el arresto de Sankoh, se sentaron las bases para estabilizar la situación; lo que no ocurriría de manera plena sino hasta el 2002.

Sólo entonces empezó a operar la CVR sierra leonesa, pero en un espacio institucional muy distinto al originalmente planeado. En efecto, la CVR debió funcionar junto a una Corte Especial creada por la ONU para juzgar a los principales perpetradores del conflicto, lo que generó una clara dificultad para cumplir el mandato de facilitar intercambios entre víctimas y victimarios, al menos en relación con los perpetradores de mayor rango. De hecho, la Corte Especial se encargó de rechazar un pedido de la CVR de organizar audiencias públicas para escuchar las perspectivas de algunos acusados bajo custodia ${ }^{8}$.

La CVR sierra leonesa debió contentarse con obtener los testimonios de perpetradores de bajo nivel; muchos de ellos víctimas, en realidad, de la práctica de reclutamiento forzoso de menores. El intento de la CVR de realizar audiencias públicas con presuntos perpetradores bajo la custodia de la Corte Especial fue rechazado por ésta con el argumento de que afectaría la integridad y la seguridad del proceso penal ${ }^{9}$.

La CVR publicó su reporte final en octubre de 2004, pero el gobierno de Ahmad Tejan Kabbah sólo se pronunció sobre éste en junio de 2005, haciendo escasa mención de una serie de recomenda-

\footnotetext{
7 "The Truth and Reconciliation Commission Act", 2000.

8 Corte Especial de Sierra Leona, "Decision on Preliminary Motions Based on Lack of Jurisdiction", 16 de marzo de 2004.

9 Corte Especial de Sierra Leona, "Decision on Appeal by the Truth and Reconciliation Commission", 28 de noviembre de 2003.
} 
ciones clave. Desde entonces, organismos de la sociedad civil sierra leonesa han producido una "tabla de resultados" ${ }^{10}$ que mide el grado de implementación de las recomendaciones de la CVR. Los resultados han sido hasta la fecha poco satisfactorios: el gobierno no se ha comprometido lo suficiente en áreas tales como el combate contra la corrupción y el manejo adecuado de recursos naturales, que sirvieron como base de la violencia.

\section{Timor Oriental}

El caso de Timor Oriental, poco conocido en América Latina, es también ilustrativo del choque entre las ilusiones políticas de reconciliación automática y las tensiones reales causadas por el mantenimiento de la impunidad. En ese pequeño país, independizado de Indonesia luego de 24 años de brutal ocupación, la ONU creó una "Comisión de Acogimiento, Verdad y Reconciliación" que operó cerca de cuatro años reconstruyendo la historia de las atrocidades cometidas por Indonesia y sus colaboradores. Al mismo tiempo, la ONU estableció Salas judiciales y una unidad especial de fiscalía para tratar crímenes de lesa humanidad.

La Comisión, aunque había recibido el mandato de contribuir a la reconciliación nacional, aplicó un procedimiento muy distinto al sudafricano. En efecto, el reglamento de la Comisión ${ }^{11}$ preveía la aplicación de mecanismos comunales de reconciliación entre víctimas y victimarios, pero los reducía solamente al caso de individuos que hubiesen cometido ofensas menores, a la par que excluía expresamente la aplicación de tales medidas a quienes resultaren sospechosos de haber cometido crímenes de lesa humanidad. De este modo, durante su trabajo, la Comisión propició la reintegración de más de 1.500 individuos en sus comunidades ${ }^{12}$. La unidad fiscal especial, establecida por la ONU al mismo tiempo que la Comisión, se encargaba de verificar que los procesos de reconciliación comunal fuesen efectivamente aplicados sólo a personas sobre las que no cupiera sospecha de participación en crímenes de lesa humanidad.

Al cabo de su mandato, la Comisión presentó su Informe Final, titulado “¡Basta!”13, al Presidente de la República -el antiguo líder guerrillero "Xanana" Gusmão-, en octubre de 2005. El informe reconstruyó penosamente múltiples patrones de violación de derechos humanos, incluyendo vívidas descripciones proporcionadas por los sobrevivientes, y propuso una serie de recomendaciones, entre ellas, la continuación de los procesos penales impulsados por la ONU contra perpetradores indonesios y la búsqueda de reparaciones para las víctimas de mayor urgencia.

La respuesta de "Xanana", sin embargo, no estuvo a la altura de las expectativas de las víctimas. En un discurso ante el parlamento ${ }^{14}$, Gusmão consideró que el Informe estaba plagado de un "grandioso idealismo" que, objetivamente, se oponía a la política de reconciliación con Indonesia seguida por los líderes timoreses. Gusmão se negó a apoyar las exigencias de justicia de “¡Basta!” y consideró que la recomendación de exigir reparaciones de los países que habían colaborado con la ocupación indonesia era una irresponsabilidad. Tanto frente al parlamento como frente al Consejo de Seguridad un par de meses después ${ }^{15}$, Gusmão consideró que una política de justicia como la exigida por la Comisión traería "anarquía política y caos social" al país y sostuvo que

\footnotetext{
10 "TRC Recommendation Scorecard Released", Concord Times (Freetown), 11 de enero de 2006.

11 United Nations Transitional Administration in East Timor - UNTAET, "Regulation 2001-10", 13 de julio de 2001.

12 Varney, Howard y Hirst, Megan, "Justice Abandoned? An Assessment of the Serious Crimes Process in East Timor", junio de 2005.

13 "Chega!", Report of the Commission for Reception Truth and Reconciliation of Timor Leste, 31 de octubre de 2005.

14 "Speech of H.E. President Kay Rala Xanana Gusmao on the Occasion of the Handing Over of the Final report of the CAVR to the National Parliament", 28 de noviembre de 2005.

155351 Período de sesiones del Consejo de Seguridad, S/ PV.5351, 23 de enero de 2006.
} 
-por el contrario-Timor e Indonesia debían seguir las recomendaciones del Arzobispo Desmond Tutu hacia un camino de justicia restaurativa.

De hecho, la oposición de Gusmão a “ßBasta!" Ilevó a su gobierno a mostrar escaso entusiasmo en la distribución del informe. El texto no sería presentado a la población timoresa, en todo o en parte, hasta julio de 2006; desgraciadamente tarde pues -para entonces- la anarquía y el caos ya se habían adueñado del país, no por conocimiento, sino por desconocimiento de la verdad histórica. Una disputa entre veteranos de la resistencia anti indonesia, que se alimentaba de leyendas sobre qué grupo sufrió más durante la ocupación, constituyó el marco para una conflagración general al interior del ejército y la policía timoresas, que escaló hasta derrocar al gobierno y motivar una ola de destrucción que recordó los peores momentos de la presencia indonesia.

La autoridad sólo se estabilizó relativamente con la presencia de tropas internacionales y la creación de un nuevo gobierno, encabezado por el primer ministro José Ramos Horta, quien debió reconocer el papel de la verdad histórica como un instrumento de prevención y no de reactivación de conflictos: "Debemos utilizar sus grandiosas enseñanzas para entender mejor las crisis del presente y para prevenir futuras crisis"16. Desde entonces, el gobierno timorés -aunque no ha actuado a favor de la implementación de sus recomendaciones- ha retirado sus reservas respecto a la más amplia diseminación del informe.

\section{Esfuerzos ilegítimos}

Otra importante señal de la práctica que se puede apreciar en los últimos años es el fracaso de comisiones planteadas como un calco radical de la experiencia sudafricana o como un claro intento de reeditar la noción de un canje de derechos. Dos casos merecen particular atención: la sentencia de la Corte Constitucional de Indonesia, anulando la ley que creaba una Comisión de la Verdad y Reconciliación en ese país, y la disolución de la Comisión de la Verdad y Reconciliación de la República Democrática del Congo.

\section{Indonesia}

En septiembre de 2004, la Cámara de Representantes de Indonesia aprobó la ley 27/2004 estableciendo una CVR para enfrentar el legado de casi seis décadas de violaciones de derechos cometidas a lo largo de la historia independiente del país. La ley era el resultado de varios años de debate parlamentario, puesto que la noción de una Comisión de la Verdad había sido planteada desde el momento mismo de la caída del régimen de Suharto en 1998 y -naturalmente- había sufrido desde entonces una serie de transformaciones.

El primer esfuerzo de crear una Comisión de la Verdad para Indonesia fue la mera traducción al idioma nacional de la legislación sudafricana estableciendo la CVR de ese país. La lógica era, pues, transparente: ¿por qué no aplicar en Indonesia lo que había funcionado en Sudáfrica? Pese a las objeciones de grupos de víctimas y de especialistas que alertaban contra la traslación mecánica del instituto sudafricano, diversos actores indonesios impulsaron el proyecto.

En el camino legislativo, sin embargo, la noción de amnistía condicional sufrió una metamorfosis que la haría irreconocible incluso para su linaje sudafricano. En primer lugar, la ley relajó notoriamente las condiciones de cooperación con la justicia necesarias para postular a una amnistía.

16 "Address by Dr José Ramos-Horta at his swearing in ceremony as Prime Minister of the Democratic Republic of TimorLeste", 10 de julio de 2006. 
En segundo lugar, creó un instrumento de presión sobre las víctimas para perdonar expresamente a los perpetradores.

En efecto, donde el esquema sudafricano obligaba al perpetrador a confesar la verdad, demostrar motivación política para sus actos y proporcionalidad entre el crimen y su motivación, la ley indonesia prescindía de todos estos elementos y se conformaba con exigir del perpetrador un "pedido de perdón" dirigido hacia la víctima. En un giro aún más problemático, la ley planteaba que si la víctima otorgaba su perdón, el perpetrador recibiría de manera automática la amnistía solicitada; pero la situación inversa no era posible: la negación del perdón no implicaba la pérdida del beneficio, pues la Comisión podría de todas formas juzgar la confesión del perpetrador por sus méritos y recomendar una amnistía. No obstante, lo más grave del esquema indonesio consistía en la facultad de la Comisión de recomendar reparaciones sólo para aquellas víctimas en cuyos casos los perpetradores hubieran recibido el beneficio de una amnistía. Es decir, las víctimas que rehusaban perdonar se pondrían a sí mismas en riesgo de perder su derecho a la reparación; por no decir nada de aquéllas sin perpetrador conocido.

Pese a contar con el marco legal para hacerlo, el gobierno indonesio no estableció la Comisión, lo que permitió a un amplio grupo de organismos defensores de los derechos humanos presentar una demanda de constitucionalidad contra la ley. En diciembre de 2006, la Corte Constitucional ${ }^{17}$ consideró que tres artículos clave de la ley violaban las garantías de derechos humanos contenidas en la Constitución nacional y que tal vicio anulaba el conjunto de la legislación.

Para la Corte, la provisión de amnistías frente a las más graves violaciones de derechos humanos constituyó una directa negación de garantías constitucionales básicas y de obligaciones internacionales de Indonesia, así como una violación adicional de los derechos de las víctimas. Además, el prerrequisito de una amnistía previa al perpetrador para otorgar reparaciones a las víctimas negaba el principio constitucional de igual protección legal para todos los ciudadanos y discriminaba contra aquéllos cuyo perpetrador no pudiera ser identificado o no recibiera una amnistía. A su vez, anulaba principios afirmados universalmente respecto a la obligación de reparar.

Dentro de este escenario, y en ausencia de una comisión oficial, numerosas organizaciones de la sociedad civil indonesia han lanzado sus propios instrumentos de búsqueda de la verdad y ha crecido el interés en "comisiones extraoficiales de la verdad", tales como las animadas por iglesias cristianas en diversos países de América Latina ${ }^{18}$.

\section{República Democrática del Congo}

Otro duro fracaso de políticas de impunidad articuladas a través de comisiones de la verdad ocurrió en la República Democrática del $\operatorname{Congo}^{19}$, donde los diálogos de Sun City (Sudáfrica) establecieron la creación de una CVR como parte de los acuerdos de paz ${ }^{20}$. La CVR así creada, a imitación de Sudáfrica, fue defendida de toda demanda judicial nacional mediante su incorporación en la nueva Constitución.

17 Corte Constitucional de Indonesia, Decisión 006/PUU-IV/2006, 7 de diciembre de 2006.

18 Ver, entre otras: Arquidiócesis de Sao Paulo, "Brasil: Nunca Mais", 1985; Servicio Paz y Justicia-Uruguay, "Uruguay: Nunca más", 1989; Arzobispado de Guatemala, "Guatemala: Nunca más", 1998.

19 Borello, Federico, "A First Few Steps. The Long Road to a Just Peace in the Democratic Republic of the Congo", octubre de 2004

20 Resolución № 20, Sobre la creación de una comisión de la verdad y reconciliación, diciembre de 2002. Artículos 154 160 de la Constitución de la República Democrática del Congo. 
Uno de los poderes centrales de la $\mathrm{CVR}^{21}$ constituía la posibilidad de otorgar amnistías para quienes confesaran crímenes cometidos "con motivación política" repitiendo, de este modo, uno de los principios aplicados por la comisión sudafricana. Al mismo tiempo, el mandato negaba que crímenes de lesa humanidad y el delito de genocidio fuesen candidatos para la amnistía, aunque precisamente ese tipo de crímenes se encontraba bajo la jurisdicción de la Comisión. Esta contradicción, potencialmente seria, nunca tuvo que ser resuelta porque la Comisión, como veremos, jamás funcionó de manera estable.

La Comisión congolesa, además, debió enfrentar desde el primer momento la más dura oposición de la sociedad civil en virtud de su composición: cada uno de los partidos integrantes del diálogo de paz en Sudáfrica aportó miembros para la CVR, sin mecanismo alguno de consulta social. Como resultado, incluyó a personas directamente acusadas de haber dirigido matanzas durante el conflicto armado interno. El rechazo masivo de la sociedad civil a la Comisión, desde su establecimiento en 2004, resultó - de un lado- en su aislamiento internacional y su completa falta de acceso a financiamiento externo y -de otro- en la más cerrada falta de cooperación de parte de las víctimas y organismos representativos.

La agonía de una comisión privada de recursos y aislada de su base natural -las víctimas- llegó a su fin con las primeras elecciones democráticas postacuerdos de paz en noviembre de 2006. Los acuerdos de Sun City estipulaban este momento como el fin de la transición y de los trabajos de la CVR, que debía presentar entonces un reporte definitivo. La CVR, incapaz de presentar dicho reporte, se consideró disuelta. Aún así, su presidente, el obispo Jen-Luc Kuye-Ndondo, fue elegido congresista en tales elecciones y ha continuado Ilamando al reestablecimiento de la Comisión.

\section{Identificando tendencias}

Diversos fenómenos recientes en el campo de la justicia transicional parecen apuntar, como esta somera revisión indica, a un análisis menos "ilusionado" y más realista de las capacidades de las comisiones de la verdad para cumplir un rol constructivo en la ruta de la reconciliación nacional. Las lecciones de la práctica Ilaman a una mirada sobria sobre el legado de la comisión sudafricana: pese a su enorme fuerza inspiradora, su legado y su debilidad se originan en el mismo mecanismo que hizo intercambiables el derecho a la verdad y el derecho al remedio judicial. Pero las experiencias recientes parecen inclinar la balanza del juicio histórico en contra del celebrado mecanismo sudafricano, incluso dramáticamente en los foros públicos de ese país.

Los mitos inspiradores de distintas políticas de justicia transicional, en tanto simplificaciones extremas, muestran sus debilidades. El "mito español" del tiempo curador de las heridas se ha visto cuestionado intensamente con el renovado activismo de los descendientes de las víctimas del franquismo. Pero en el otro extremo, el de la fe en una verdad capaz de reconciliar en forma automática, el "mito sudafricano" también se ha visto afectado.

La tendencia que más claramente se expresa en el rechazo a la reedición del mecanismo de "verdad por amnistía" es la noción de políticas integrales de justicia transicional, en las que distintos mecanismos de búsqueda de la verdad, justicia penal y reparación encuentren mejores formas de interacción. Las comisiones de Sierra Leona y Timor Oriental debieron trabajar en relación con tribunales híbridos, y otras comisiones recientes como las de Perú, que culminó en 2003, y la de Paraguay, que continúa en actividad, se entienden como auxiliares -no opuestas-a la tarea de organismos judiciales.

21 Ley del 30 de julio de 2004. 
El fracaso de instancias predicadas en el mito de Sudáfrica no quiere decir que las comisiones de la verdad -desprovistas de un mito motivador- hayan dejado de resultar atractivas. En Liberia, el gobierno de la Sra. Ellen Sirleaf-Johnson reformó una comisión creada con un mandato muy similar a (y una composición tan criticada como) la comisión congolesa, y estableció un nuevo organismo con un mandato meticulosamente revisado y consultado con la sociedad civil y la comunidad internacional. En la misma República Democrática del Congo, un "comité de seguimiento" de la sociedad civil y la comunidad internacional evalúa la posibilidad de una nueva legislación para una comisión corregida.

Adicionalmente, la sociedad civil ha llevado su activismo a niveles de sofisticación cada vez mayores. Inspiradas en un "mito alternativo", el de los informes extraoficiales, entre ellos el celebrado informe guatemalteco ${ }^{22}$, diversas instituciones de la sociedad civil han lanzado esfuerzos parciales o locales de búsqueda de la verdad. Sólo en Colombia, en medio de un controversial proceso de desmovilización de grupos paramilitares, se pueden contar una veintena de iniciativas no oficiales. Propuestas similares se registran en Indonesia, Irak, Serbia y Sri Lanka.

La comunidad internacional, en particular los organismos de la ONU, también han ganado en sofisticación y su apoyo a comisiones de la verdad en situaciones postconflicto ha perdido el automatismo que sugería su actuación en años recientes. Con la adopción de lineamientos por parte de la Comisión de Derechos Humanos y del Secretario General, la ONU se ha vuelto más cautelosa en su tratamiento de la búsqueda de verdad en procesos transicionales. En Burundi, luego de negociaciones con el gobierno, el Consejo de Seguridad ${ }^{23}$ acaba de establecer una nueva oficina que incluye el mandato de apoyar todo esfuerzo de combate contra la impunidad, incluyendo la creación de una comisión de la verdad y un tribunal especial estructurado al modo de los tribunales híbridos de Sierra Leona y Timor Oriental. En Nepal, pese a los espectaculares sucesos que culminaron en el acuerdo de paz $^{24}$ entre la guerrilla maoísta y los partidos de la oposición pacífica a la monarquía, la Alta Comisionada de Derechos Humanos de la ONU, Sra. Louise Arbour, ha llamado al enjuiciamiento de los responsables de los más graves crímenes ${ }^{25}$, y todo indica que el escaso avance hacia una comisión se debe a la reluctancia de la ONU a apoyar su creación apresurada, mal diseñada o contrapuesta a los derechos de las víctimas.

No es posible suponer que el entusiasmo por las comisiones de la verdad se extinga muy pronto. Sin embargo, es probable que la mayor conciencia de sus limitaciones enerve las ilusiones de algunos actores políticos. Si no es viable lograr un escenario al estilo de Sudáfrica o si aquél escenario no era tan real como se cree, ¿cuál sería la ventaja de establecer una comisión? ¿Sería suficiente motivación la de generar mejores condiciones para manejar los tiempos de la transición en tanto se debilita el poder autoritario subsistente?

No es fácil responder a estas preguntas ahora. El mayor realismo que se precisa para reconocer las capacidades y limitaciones del instrumento analizado, todavía dista de materializarse por completo. Es previsible que el drama de un proceso y un ritual que efectúe una sólida ruptura simbólica con el pasado siga concitando la imaginación de muchas personas en diversas latitudes. La pregunta que queda abierta es si la superación de ciertos presupuestos poco realistas permitirá el surgimiento de una tercera, más sofisticada, "generación" de comisiones de la verdad.

\footnotetext{
22 Ver nota $\mathrm{N}^{\circ} 18$ del presente trabajo.

23 Resolución No 1719/2006, 26 de octubre de 2006.

24 "Comprehensive Peace Agreement", 26 de noviembre de 2006.

25 "L'ONU incite le Népal à juger les auteurs des violations des droits de I'homme durant le conflit armé", Le Monde, 26 de enero de 2007.
} 